\title{
Solving the Multiobjective Fractional Transportation Problem through the Neutrosophic Goal Programming Approach
}

\author{
C. Veeramani, ${ }^{1}$ S. A. Edalatpanah $\mathbb{D D}^{2},{ }^{2}$ and S. Sharanya ${ }^{1}$ \\ ${ }^{1}$ Department of Applied Science (Mathematics), PSG College of Technology, Coimbatore 641 004, Tamil Nadu, India \\ ${ }^{2}$ Department of Applied Mathematics, Ayandegan Institute of Higher Education, Tonekabon, Iran
}

Correspondence should be addressed to S. A. Edalatpanah; saedalatpanah@gmail.com

Received 8 June 2021; Revised 31 July 2021; Accepted 10 August 2021; Published 30 August 2021

Academic Editor: Dragan Pamučar

Copyright ( 2021 C. Veeramani et al. This is an open access article distributed under the Creative Commons Attribution License, which permits unrestricted use, distribution, and reproduction in any medium, provided the original work is properly cited.

Nowadays, the transportation problem is a multiobjective decision-making problem. It involves deciding to determine the ideal transportation setup that matches the decision maker's preferences while taking into account competing objectives/criteria such as transportation cost, transportation time, and environmental and social concerns. This study presents a general framework of the multiobjective fractional transportation problem (MOFTP) to deal with such complex scenarios. This paper's major goal is to propose a solution methodology to solve the MOFTP based on a neutrosophic goal programming (NGP) approach. By obtaining the optimal compromise solution using three memberships, namely, truth membership, indeterminacy membership, and falsity membership, the suggested technique gives a novel insight into solving the MOFTP. A real-world problem such as selling wind turbine blades' problem and a numerical example are used to demonstrate the efficacy and superiority of the proposed method.

\section{Introduction}

Transportation problems (TPs) are formulated to transport different types of products to different locations for lower cost, lower transport time, lower production cost, and more. For example, suppose a distribution center seeks to determine the transportation plan to transport identical goods from $M$ sources to $N$ locations. In addition, each source has materials to deliver to different locations, and each destination has a forecast request of products to be sourced from sources. Thus, when a TP problem is proposed, it tries to determine the optimal volumes to carry from each source to each destination by minimizing the cost of production, transportation cost, and delivery time. Hitchcock invented the traditional transportation problem [1]. Based on the primal simplex transportation technique, Dantzig and Thapa [2] proposed the simplex method to solve the TP. Recently, Ezekiel and Edeki [3] developed a new method to solve the TP which is called modified Vogel's approximation method.

Due to numerous features of the decision maker as well as real-life settings for an industrial problem, several objective functions such as minimization of transportation cost and transportation time are generally considered in a real-life transporting system. Transportation problems have involved more than one objective function, called multiobjective transportation problems (MOTPs), which played a predominant role in production planning, supply chain management, etc. Hence, solving the MOTPs is important in reallife applications. Many authors have studied multiobjective transportation issues in depth. Amaliah et al. [4] suggested finding the initial basic feasible solution based on the heuristic method. Karagul and Sahin [5] established the Karagul-Sahin approximation method to arrive at an efficient initial basic feasible solution while dealing with the TP. Instead of the optimal solution, the optimal compromise solution or effective solution is found for the MOTPs. Goal programming (GP) [6], utility approaches [7-9], collaborative procedure [10], fuzzy programming approaches [11-16], and intuitionist fuzzy programming approaches [17-19] have been established to find a compromise solution to the MOTP. Zimmermann proposed a novel method for solving ill-conditioned multicriteria optimization problems based on the fuzzy programming technique. To decide the optimum solution, Lohgaonkar and Bajaj [20] developed a multiobjective 
capacitated TP and used three different types of membership functions, namely, linear, exponential, and hyperbolic. Later, Gupta and Bari [21] implemented the multiobjective capacitated TP with mixed constraints. Nomani et al. [22] established a new weighted GP method for the MOTP that included some priority sets for each objective function. Pramanik and Banerjee [23] proposed a chance-constrained capacitated MOTP with two fuzzy goals, and a consensus solution was found. Gupta et al. established a fuzzy goal programming framework for solving the MOTP based on minimizing the negative deviational variables.

Some cases of objectives for transportation problems belonging to the fractional programming category may include the minimization of total actual cost/standard cost, actual time/ standard time, actual deterioration/standard deterioration, and risk assets/capital [24]. Sadia et al. [25] proposed a compromise solution to a MOFTP with mixed constraints by using fuzzy programming and lexicographic GP with minimum distance methods. Costa [26] proposed a new method for optimizing a weighted sum of linear fractional objective functions. With a given weight vector, this technique produces only one nondominated solution to the MOLFP.

Fuzzy set theory is vividly used to predict the performance values of securities in an uncertain environment. However, the fuzzy goal programming (FGP) focuses only on the degree of truth membership, and it does not take into account the nonmembership and indeterminacy. Atanassov [27] developed the intuitionistic fuzzy set theory. The intuitionistic fuzzy goal programming (IFGP) has been vividly applied in decision-making problems [28]. IFS has also been used by several researchers to solve various forms of transportation problems $[17,18]$. IFS takes into account both degree of truth and degree of falsity but does not find indeterminacy. So, it fails to deal with indeterminacy existing in the real world. To overcome these drawbacks of the fuzzy set and IFS, neutrosophic set (NS) has been used in an uncertain environment.

The NS is an extent or generalization of the IFS. It represents real-world problems effectively and efficiently by considering all aspects of decision situations [29]. Nafei and Nasseri [30] analyzed neutrosophic integer programming problems. Edalatpanah [31] developed a nonlinear framework for neutrosophic linear programming. Furthermore, Rizk-Allah et al. [32] developed a compromise solution framework for the MOTP based on the neutrosophic environment. Ye [33] demonstrated some simple neutrosophic number operations as well as a neutrosophic function and then constructed a neutrosophic number linear programming (NNLP) system to handle neutrosophic number optimization problems. Recently, Ahmad [34] solved multiobjective optimization based on the robust neutrosophic programming technique. Hence, in this paper, a new compromise solution framework is developed for solving the MOFTPs based on the neutrosophic goal programming approach. In the neutrosophic context, the proposed neutrosophic programming method is developed by expanding the Zimmermann principles [35]. By reaching the optimum compromise solution combining three memberships, namely, truth membership, indeterminacy membership, and falsity membership, the suggested framework provides new insight into the neutrosophic environment in the MOFTP. To the best of our knowledge, there exists no literature taking into consideration to solve the MOFTP on the neutrosophic goal programming approach.

The major contributions are outlined as follows:

The MOFTP model has been developed to assist decision makers in overcoming the problems they confront when making real-life judgments

The best compromise solution is determined by applying a neutrosophic programming framework to the MOFTP

An illustration example and a case study problem are used to demonstrate the feasibility and validity of the NGP technique

The efficiency of the proposed method is proved through a comparative study

The rest of the work of this paper is organized as follows: Section 2 describes the mathematical formulation of the MOFTP and basic concepts of the neutrosophic set. Section 3 develops the neutrosophic goal programming approach for the MOFTP. Section 4 analyzes advantages and limitations of the proposed method. Section 5 provides the numerical example and case study problem for validating the theoretical concepts. Section 6 deals with results and discussion. Finally, Section 7 discusses the conclusion and presents the scope for future research.

\section{Mathematical Description of the MOFTP and Neutrosophic Set}

In this section, we describe the general form of the MOFTP and neutrosophic set. For modeling the MOFTP, we use the notations such as variables, parameters, and indexes given in Table 1.

The generalized mathematical model of the MOFTP is given as

$$
\begin{aligned}
& \operatorname{Min} Z_{k}=\frac{N_{k}(x)}{D_{k}(x)}=\frac{\sum_{i=1}^{m} \sum_{j=1}^{n} n_{i j}^{(k)} x_{i j}}{\sum_{i=1}^{m} \sum_{j=1}^{n} d_{i j}^{(k)} x_{i j}}, \quad k=1,2, \ldots, K, \\
& \text { subject to } \sum_{j=1}^{n} x_{i j} \leq a_{i}, \quad i=1,2, \ldots, m, \\
& \qquad x_{i=1}^{m} x_{i j} \geq b_{j}, \quad j=1,2, \ldots, n, \\
& x_{i j} \geq 0 \quad i=1,2, \ldots, m, j=1,2, \ldots, n .
\end{aligned}
$$

The constraint ensures the limitation on the products to be transported because of some finite availability, and the demand constraint ensures the fulfillment of the demand of the product at each destination, respectively. 
TABLE 1: List of symbols and observation.

\begin{tabular}{lc}
\hline & List of symbols and observation \\
\hline$x_{i j}$ & Amount of the product transported from source $i$ to destination $j$ \\
$n_{i j}^{(k)}$ & Coefficient vector for the numerator of the $k^{\text {th }}$ objective from source $i$ to destination $j$ \\
$d_{i j}^{(k)}$ & Coefficient vector for the denominator of the $k^{\text {th }}$ objective from source $i$ to destination $j$ \\
$a_{i}$ & Amount of the product available at source $i$ \\
$b_{i}$ & Demand of the product available at destination $j$ \\
$\xi(x)$ & Truth membership function \\
$\psi(x)$ & Indeterminacy membership function \\
$\zeta(x)$ & Falsity membership function \\
FGP & Fuzzy goal programming \\
FS & Fuzzy set \\
GP & Goal programming \\
IFGP & Intuitionistic fuzzy goal programming \\
IFS & Intuitionistic fuzzy set \\
MOFTP & Multiobjective fractional transportation problem \\
MOLFP & Multiobjective linear fractional problem \\
MOTP & Multiobjective transportation problem \\
NGP & Neutrosophic goal programming \\
NS & Neutrosophic set \\
TP & Transportation problem \\
\hline
\end{tabular}

Definition 1 (efficient solution; see [36]). Any feasible solution $X^{*}=\left\{x_{i j}^{*}\right\} \in$ feasible solution set $S$ is said to be an efficient solution of (1) if there is no other feasible solution $X=\left\{x_{i j}\right\} \in S$ such that

$$
\begin{aligned}
& \sum_{i=1}^{m} \sum_{j=1}^{n} C_{i j}^{k} x_{i j} \leq \sum_{i=1}^{m} \sum_{j=1}^{n} C_{i j}^{k} x_{i j}^{*}, \quad \forall k, k=1,2, \ldots, K, \\
& \sum_{i=1}^{m} \sum_{j=1}^{n} C_{i j}^{k} x_{i j}<\sum_{i=1}^{m} \sum_{j=1}^{n} C_{i j}^{k} x_{i j}^{*}, \quad \text { for at least one } k, k=1,2, \ldots, K .
\end{aligned}
$$

Definition 2 (compromise solution; see [36]). A feasible solution $X^{*}=\left\{x_{i j}^{*}\right\} \in S$ is called a preferred compromise solution for problem (1) iff $X^{*} \in$ efficient set of solutions and $Z\left(X^{*}\right) \leq Z(X)$, where $X \in S$.

Definition 3 (neutrosophic set; see [37]). Let $X$ be a universe discourse such that $x \in X$; a neutrosophic set $U$ in $X$ is defined by three membership functions, namely, the degree of membership function (or) truth membership function $\left(\xi_{U}(x)\right)$, the degree of indeterminacy (or) indeterminacy $\left(\psi_{U}(x)\right)$, and the degree of nonmembership (or) falsity $\left(\xi_{U}(x)\right)$, and is denoted by the following form:

$$
U=\left\{\left\langle x, \xi_{U}(x), U(x), U(x)\right\rangle: \quad x \in X\right\},
$$

where $\xi_{U}(x), \psi_{U}(x)$, and $\zeta_{U}(x)$ are real standard or nonstandard subsets belonging to $] 0^{-}, 1^{+}[$, and they are also given as $\left.\xi_{U}: X \longrightarrow 0^{-}, 1^{+}\right], \psi_{U}: X \longrightarrow 0^{-}, 1^{+}$, and $\left.\zeta_{U}: X \longrightarrow\right] 0^{-}, 1^{+}[$. There is no restriction on the sum of $\xi_{U}(x), \psi_{U}(x)$, and $\zeta_{U}(x)$, so we have

$$
0^{-} \leq \sup \xi_{U}(x)+\sup \psi_{U}(x)+\sup \zeta_{U}(x) \leq 3^{+} .
$$

Definition 4 (single-valued neutrosophic set (SVNS); see [37]). A single-valued neutrosophic set $U$ over the universe of discourse $X$ is defined as

$$
U=\left\{\left\langle x, \xi_{U}(x), \psi_{U}(x), \zeta_{U}(x)\right\rangle: \quad x \in X\right\},
$$

where $\xi_{U}(x), \psi_{U}(x)$, and $\zeta_{U}(x) \in[0,1]$ and $0 \leq \xi_{U}(x)+\psi_{U}(x)+\zeta_{U}(x) \leq 3$ for each $x \in X$.

The procedures using the neutrosophic optimization approach for solving the MOFTP are explained in Section 3.

\section{Neutrosophic Goal Programming}

A new strategy to solve the MOFTP based on neutrosophic goal programming is provided in this section. The proposed methodology is based on a neutrosophic extension of Zimmermann's principles [35]. The presented neutrosophic compromise programming technique offers a new perspective on dealing with indeterminacy in optimization problems, with the goal of simultaneously optimizing the degrees of truth (satisfaction), falsity (dissatisfaction), and indeterminacy (satisfaction to some extent) of a neutrosophic decision. Bellman and Zadeh [38] introduced three concepts for the fuzzy set, fuzzy decision $(D)$, fuzzy goal $(G)$, and fuzzy constraints $(C)$, and implemented these concepts in many applications of decision-making under fuzziness. The fuzzy decision is defined as follows:

$$
D=G \cap C \text {. }
$$

Accordingly, the neutrosophic decision set $D_{N}$, a conjunction of neutrosophic objectives and constraints, is defined:

$$
D_{N}=\left(\bigcap_{k=1}^{K} G_{k}\right)\left(\bigcap_{i=1}^{m} C_{i}\right)=\left(x, \xi_{D}(x), \psi_{D}(x), \zeta_{D}(x)\right),
$$

where 


$$
\begin{aligned}
& \xi_{D}(x)=\min \left\{\begin{array}{c}
\xi_{G_{1}}(x), \xi_{G_{2}}(x), \ldots, \xi_{G_{K}}(x) ; \\
\xi_{C_{1}}(x), \xi_{C_{2}}(x), \ldots, \xi_{C_{m}}(x)
\end{array}\right\}, \quad \text { for all } x \in X \\
& \psi_{D}(x)=\min \left\{\begin{array}{l}
\psi_{G_{1}}(x), \psi_{G_{2}}(x), \ldots, \psi_{G_{K}}(x) ; \\
\psi_{C_{1}}(x), \psi_{C_{2}}(x), \ldots, \psi_{C_{m}}(x)
\end{array}\right\}, \quad \text { for all } x \in X \text {, } \\
& \zeta_{D}(x)=\min \left\{\begin{array}{c}
\zeta_{G_{1}}(x), \zeta_{G_{2}}(x), \ldots, \zeta_{G_{K}}(x) ; \\
\zeta_{C_{1}}(x), \zeta_{C_{2}}(x), \ldots, \zeta_{C_{m}}(x)
\end{array}\right\} \text {, for all } x \in X \text {, }
\end{aligned}
$$

where $\xi_{D}(x)$ is the truth membership function, $\psi_{D}(x)$ is the indeterminacy membership function, and $\zeta_{D}(x)$ is the falsity membership function of neutrosophic decision set $D_{N}$.

To formulate the membership functions for the MOFTP, the bounds for each objective function are determined. The lower and upper bounds for each objective are denoted by $Z_{k}$ and $\overline{Z_{k}}$ that are calculated as follows: each objective is optimized as a single objective subjected to the problem constraints. By solving $K$ objectives individually, we obtain $K$ solutions, $X^{1}, X^{2}, \ldots, X^{K}$. Afterward, these solutions are substituted in each objective to explore the bounds for each objective as follows:

$$
\begin{aligned}
& \overline{Z_{k}}=\max \left\{Z_{k}(x)\right\}_{k=1}^{K}, \\
& \underline{Z_{k}}=\min \left\{Z_{k}(x)\right\}_{k=1}^{K} .
\end{aligned}
$$

Then, the bounds for the neutrosophic environment are calculated as follows:

$$
\begin{aligned}
& \bar{Z}_{k}^{\xi}=\overline{Z_{k}}, \underline{Z_{k}}=\underline{Z_{k}}, \text { for the truth membership, } \\
& \bar{Z}_{k}^{\psi}=\underline{Z_{k}}+s_{k}\left(\bar{Z}_{k}^{\xi}-\underline{Z_{k}}\right), \underline{Z_{k}}=\underline{Z_{k}}, \text { for the indeterminacy membership, } \\
& \bar{Z}_{k}^{\zeta}=\bar{Z}_{k}^{\xi}, \underline{Z_{k}}=\underline{Z_{k}}+t_{k}\left(\bar{Z}_{k}^{\xi}-\underline{Z_{k}}\right), \text { for the falsity membership, }
\end{aligned}
$$

where $t_{k}$ and $s_{k}$ are predetermined real numbers in $(0,1)$.

According to the above bounds, the membership functions can be introduced as follows:

$$
\begin{aligned}
& \xi_{k}\left(Z_{k}(x)\right)= \begin{cases}1, & \text { if } Z_{k}(x)<\underline{Z_{k}}, \\
1-\frac{\left(\sum_{i=1}^{m} \sum_{j=1}^{n} n_{i j}^{(k)} x_{i j} / \sum_{i=1}^{m} \sum_{j=1}^{n} d_{i j}^{(k)} x_{i j}\right)-\underline{Z_{k}}}{\overline{Z_{k}}-\underline{Z_{k}}}, & \text { if } \underline{\underline{Z_{k}}} \leq Z_{k}(x) \leq \overline{Z_{k}}, \\
0, & \text { if } Z_{k}(x)>\overline{Z_{k}},\end{cases} \\
& \psi_{k}\left(Z_{k}(x)\right)= \begin{cases}1, & \text { if } Z_{k}(x)<\underline{Z_{k}}, \\
1-\frac{\left(\sum_{i=1}^{m} \sum_{j=1}^{n} n_{i j}^{(k)} x_{i j} / \sum_{i=1}^{m} \sum_{j=1}^{n} d_{i j}^{(k)} x_{i j}\right)-\underline{Z_{k}}}{\bar{Z}_{k}^{\psi}-\underline{Z_{k}},} & \text { if } \underline{Z_{k}} \leq Z_{k}(x) \leq \bar{Z}_{k}^{\psi}, \\
0, & \text { if } Z_{k}(x)>\bar{Z}_{k}^{\psi},\end{cases}
\end{aligned}
$$




$$
\zeta_{k}\left(Z_{k}(x)\right)= \begin{cases}1, & \text { if } Z_{k}(x)>\overline{Z_{k}} \zeta, \\ 1-\frac{\overline{Z_{k}} \zeta-\left(\sum_{i=1}^{m} \sum_{j=1}^{n} n_{i j}^{(k)} x_{i j} / \sum_{i=1}^{m} \sum_{j=1}^{n} d_{i j}^{(k)} x_{i j}\right)}{\bar{Z}_{k}^{\zeta}-\underline{Z_{k}}}, & \text { if } \underline{Z_{k}} \leq Z_{k}(x) \leq \overline{Z_{k}} \zeta, \\ 0, & \text { if } Z_{k}(x)<\underline{Z_{k}},\end{cases}
$$

where ${\overline{Z_{k}}}^{(\cdot)} \neq Z_{k}{ }^{(\cdot)}$ for all objectives. If ${\overline{Z_{k}}}^{(\cdot)}=Z_{k}{ }^{(\cdot)}$ for any membership, then the value of this membership is set to 1 . Using (11)-(13) and the Bellman and Zadeh [38] principle, the neutrosophic optimization model of the MOFTP can be stated as follows:

$\operatorname{Max} \operatorname{Min}_{k=1,2, \ldots, K} \xi_{k}\left(Z_{k}(x)\right)$
$\operatorname{Min} \operatorname{Max}_{k=1,2, \ldots, K} \zeta_{k}\left(Z_{k}(x)\right)$
$\operatorname{Max} \operatorname{Min}_{k=1,2, \ldots, K} \psi_{k}\left(Z_{k}(x)\right)$

subject to

$$
\begin{aligned}
& \sum_{j=1}^{n} x_{i j} \leq a_{i}, \quad i=1,2, \ldots, m, \\
& \sum_{i=1}^{m} x_{i j} \geq b_{j}, \quad j=1,2, \ldots, n, \\
& x_{i j} \geq 0, \quad i=1,2, \ldots, m, j=1,2, \ldots, n .
\end{aligned}
$$

By using auxiliary parameters, problem (14) can be transformed to the following problem:

$$
\begin{aligned}
& \operatorname{Max} \alpha, \operatorname{Max} \gamma, \operatorname{Min} \beta \\
& \xi_{Z_{k}}(x) \geq \alpha, \psi_{Z_{k}}(x) \geq \gamma, \zeta_{Z_{k}}(x) \geq \beta, \\
& \sum_{j=1}^{n} x_{i j} \leq a_{i}, \quad i=1,2, \ldots, m, \\
& \text { subject to } \sum_{i=1}^{m} x_{i j} \geq b_{j}, \quad j=1,2, \ldots, n \text {, } \\
& x_{i j} \geq 0, \quad i=1,2, \ldots, m, j=1,2, \ldots, n \\
& \alpha \geq \gamma, \alpha \geq \beta, \alpha+\gamma+\beta \leq 3, \quad \alpha, \gamma, \beta \in[0,1] \text {, } \\
& k=1,2, \ldots, K \text {. }
\end{aligned}
$$

$\operatorname{Max} \alpha-\beta+\gamma$,

$$
\begin{aligned}
& \frac{\sum_{i=1}^{m} \sum_{j=1}^{n} n_{i j}^{(k)} x_{i j}}{\sum_{i=1}^{m} \sum_{j=1}^{n} d_{i j}^{(k)} x_{i j}}+\left(\bar{Z}_{k}^{\xi}-\underline{\underline{Z}} \underline{\underline{Z}}\right) \alpha \leq \bar{Z}_{k}^{\xi}, \\
& \frac{\sum_{i=1}^{m} \sum_{j=1}^{n} n_{i j}^{(k)} x_{i j}}{\sum_{i=1}^{m} \sum_{j=1}^{n} d_{i j}^{(k)} x_{i j}}+\left(\bar{Z}_{k}^{\psi}-\underline{Z_{k}}\right) \gamma \leq \bar{Z}_{k}^{\psi}, \\
& \frac{\sum_{i=1}^{m} \sum_{j=1}^{n} n_{i j}^{(k)} x_{i j}}{\sum_{i=1}^{m} \sum_{j=1}^{n} d_{i j}^{(k)} x_{i j}}+\left(\bar{Z}_{k}^{\zeta}-\underline{Z_{k}}\right) \beta \leq \bar{Z}_{k}^{\zeta},
\end{aligned}
$$

subject to

$$
\begin{aligned}
& \sum_{j=1}^{n} x_{i j} \leq a_{i}, \quad i=1,2, \ldots, m, \\
& \sum_{i=1}^{m} x_{i j} \geq b_{j}, \quad j=1,2, \ldots, n \\
& x_{i j} \geq 0, \quad i=1,2, \ldots, m, j=1,2, \ldots, n, \\
& \alpha \geq \gamma, \alpha \geq \beta, \alpha+\gamma+\beta \leq 3, \quad \alpha, \gamma, \beta \in[0,1], k=1,2, \ldots, K .
\end{aligned}
$$


Problem (16) can be written as

$$
\begin{aligned}
& \operatorname{Max} \alpha-\beta+\gamma \\
& \sum_{i=1}^{m} \sum_{j=1}^{n} n_{i j}^{(k)} x_{i j}+\left(\overline{Z_{k}} \overline{\underline{Z}}-\underline{\underline{Z}}\right) \alpha-\overline{Z_{k}}{ }^{\xi}\left(\sum_{i=1}^{m} \sum_{j=1}^{n} d_{i j}^{(k)} x_{i j}\right) \leq 0, \\
& \sum_{i=1}^{m} \sum_{j=1}^{n} n_{i j}^{(k)} x_{i j}+\left(\overline{Z_{k}} \psi-\underline{Z_{k}}\right) \gamma-\overline{Z_{k}} \psi\left(\sum_{i=1}^{m} \sum_{j=1}^{n} d_{i j}^{(k)} x_{i j}\right) \leq 0, \\
& \text { subject to } \sum_{i=1}^{m} \sum_{j=1}^{n} n_{i j}^{(k)} x_{i j}+\left(\overline{Z_{k}^{\zeta}}-\underline{Z_{k}}\right) \beta-\overline{Z_{k}} \zeta\left(\sum_{i=1}^{m} \sum_{j=1}^{n} d_{i j}^{(k)} x_{i j}\right) \leq 0 \text {, } \\
& \sum_{j=1}^{n} x_{i j} \leq a_{i}, \quad i=1,2, \ldots, m, \\
& \sum_{i=1}^{m} x_{i j} \geq b_{j}, \quad j=1,2, \ldots, n, \\
& x_{i j} \geq 0, \quad i=1,2, \ldots, m, j=1,2, \ldots, n . \\
& \alpha \geq \gamma, \alpha \geq \beta, \alpha+\gamma+\beta \leq 3, \quad \alpha, \gamma, \beta \in[0,1], k=1,2, \ldots, K \text {. }
\end{aligned}
$$

\section{Advantages and Limitations}

Several authors have used GP-, FGP-, and IFGP-based optimization methods for solving multiobjective optimization problems. However, the existing methodologies have many shortcomings that are addressed by using the proposed interactive neutrosophic programming methodology. The nonconstancy of the rates at which benefits from objective attainments increase, as well as the nonconstancy of the rates at which decision makers will trade off attainments, are ignored in the GP methodology. Only the degree of acceptance of an element into a feasible solution set is considered in a fuzzy programming approach. The degree of rejections of the element into the same feasible solution set, which is an integral aspect of the decision-making processes, is not taken into account. The degree of belongingness and nonbelongingness of an element within the same feasible solution set are dealt with simultaneously in intuitionistic fuzzy programming. As a result, the principal reason for the IFGP's approach is to avoid the shortcomings of the FGP. In spite of the use of the FS- and IFS-based goal programming approaches, treating vague and imprecise uncertainty in various regions continues to lag behind more realistic decision-making scenarios in which indeterminate information or neutral thinking cannot be addressed. Indeterminacy is a state of uncertainty about the values of assertions somewhere between truth and falsity. Under the truth, indeterminacy, and falsity membership functions, the suggested NGP methodology considers the quantification of marginal evaluations. The limitations of the proposed method are discussed below.

The TP is a special case of a linear programming problem since it has the unimodularity feature. When a problem has a unimodularity feature, the simplex approach can be used to find a strict integer solution. The determinant of all the square submatrices in the coefficient matrix of the constraints of problem (1) is either $0,+1$, or -1 , revealing the unimodularity property. If this condition is not met, the integer solution is not guaranteed. Moreover, the problem is not a traditional TP. Hence, integer programming is employed to solve problem (17).

\section{Numerical Example}

Consider the following transportation problem: 


$$
\begin{aligned}
& \operatorname{Min} Z_{1}=\frac{10 x_{11}+14 x_{12}+8 x_{13}+12 x_{14}+8 x_{21}+12 x_{22}+14 x_{23}+8 x_{24}+9 x_{31}+6 x_{32}+15 x_{33}+9 x_{34}}{15 x_{11}+12 x_{12}+16 x_{13}+8 x_{14}+10 x_{21}+6 x_{22}+13 x_{23}+12 x_{24}+13 x_{31}+15 x_{32}+12 x_{33}+10 x_{34}}, \\
& \operatorname{Min} Z_{2}=\frac{14 x_{11}+9 x_{12}+11 x_{13}+9 x_{14}+12 x_{21}+9 x_{22}+6 x_{23}+15 x_{24}+6 x_{31}+9 x_{32}+12 x_{33}+10 x_{34}}{12 x_{11}+14 x_{12}+7 x_{13}+17 x_{14}+6 x_{21}+11 x_{22}+13 x_{23}+10 x_{24}+9 x_{31}+15 x_{32}+12 x_{33}+16 x_{34}},
\end{aligned}
$$

subject to

$$
\begin{aligned}
& x_{11}+x_{12}+x_{13}+x_{14} \leq 15 ; \\
& x_{21}+x_{22}+x_{23}+x_{24} \leq 25 ; \\
& x_{31}+x_{32}+x_{33}+x_{34} \leq 20 ; \\
& x_{11}+x_{21}+x_{31} \geq 15 ; \\
& x_{12}+x_{22}+x_{32} \geq 25 ; \\
& x_{13}+x_{23}+x_{33} \geq 5 ; \\
& x_{14}+x_{24}+x_{34} \geq 15 ; \\
& x_{i j} \geq 0, \quad i=1,2,3,4, j=1,2,3 .
\end{aligned}
$$

The numerical problem is solved using the neutrosophic mathematical programming approach in the following steps:

Step 1: solve the above model in each objective function individually as a single-objective transportation problem subject to the same constraints (18) and the individual solution as $X_{1}=(0,0,0,15,0,25,0,0,15,0,5,0)$ and $X_{2}=(5,5,5,0,10,0,0,15,0,20,0,0)$.

Step 2: construct the payoff matrix by evaluating three objectives with the obtained three solutions.

$$
\begin{aligned}
& Z_{1} \quad Z_{2} \\
& Z_{1}\left(\begin{array}{ll}
1.3143 & 0.7035
\end{array}\right) \\
& Z_{2}\left(\begin{array}{ll}
0.6838 & 1.0299
\end{array}\right)
\end{aligned}
$$

Step 3: compute the lower and upper bounds for all objectives with the payoff matrix, which are assigned using the following formula:

$$
\begin{aligned}
& \overline{Z_{k}}=\max \left\{Z_{k}(x)\right\}_{k=1}^{2}, \\
& \underline{Z_{k}}=\min \left\{Z_{k}(x)\right\}_{k=1}^{2} .
\end{aligned}
$$

The bounds of each objective function are defined by

$$
\begin{aligned}
& 0.6838 \leq Z_{1} \leq 1.3143 \\
& 0.7035 \leq Z_{2} \leq 1.0299
\end{aligned}
$$

Step 4: define all membership functions based on the neutrosophic concept for three objectives as follows:

For the first objective $Z_{1}$ :

$$
\begin{aligned}
& \bar{Z}_{1}^{\xi}=1.3143, \underline{Z_{1}}=0.6038, \\
& \bar{Z}_{1}^{\psi}=\underline{Z_{1}}+S_{1}\left(\overline{Z_{1}} \xi \underline{\underline{Z_{1}}}\right)=0.6038+s_{1}, \underline{Z_{1}}=\underline{Z_{1}}=0.6038, \\
& \bar{Z}_{1}^{\zeta}={\overline{Z_{1}}}^{\xi}=1.3148, \underline{\underline{Z}} \underline{\underline{Z}}=\underline{Z_{1}}+t_{1}\left(\bar{Z}_{1}^{\xi}-\underline{Z_{1}}\right)=0.6038+t_{1} .
\end{aligned}
$$

Truth, indeterminacy, and falsity membership functions of the first objective $Z_{1}$ are as follows: 


$$
\begin{aligned}
& \xi_{1}\left(Z_{1}(x)\right)= \begin{cases}1, & \text { if } Z_{1}(x)<0.6038 \\
1-\frac{Z_{1}(x)-0.6038}{1.3148-0.6038}, & \text { if } 0.6038 \leq Z_{1}(x) \leq 1.3148 \\
0, & \text { if } Z_{1}(x)>1.3148\end{cases} \\
& \psi_{1}\left(Z_{1}(x)\right)= \begin{cases}1, & \text { if } Z_{1}(x)<0.6038, \\
1-\frac{Z_{1}(x)-0.6038}{s_{1}}, & \text { if } 0.6038 \leq Z_{1}(x) \leq 0.6038+s_{1}, \\
0, & \text { if } Z_{1}(x)>0.6038+s_{1},\end{cases} \\
& \zeta_{1}\left(Z_{1}(x)\right)= \begin{cases}1, & \text { if } Z_{1}(x)>1.3148, \\
1-\frac{1.3148-Z_{1}(x)}{1.3148-0.6038-t_{1},} & \text { if } 0.6038+t_{1} \leq Z_{1}(x) \leq 1.3148, \\
0, & \text { if } Z_{1}(x)<0.6038+t_{1} .\end{cases}
\end{aligned}
$$

For the second objective $Z_{2}$ :

Truth, indeterminacy, and falsity membership functions of the second objective $Z_{2}$ are as follows:

$$
\begin{aligned}
& \bar{Z}_{2}^{\xi}=1.0299, \\
& \underline{Z_{2}}=0.7035, \\
& \bar{Z}_{2}^{\psi}=\underline{\underline{Z}} \underline{\underline{Z}}+s_{2}\left(\bar{Z}_{2}^{\xi}-\underline{\underline{Z}} \underline{\underline{Z}}\right)=0.7035+s_{2}, \\
& \underline{Z_{2}}=\underline{Z_{2}}=0.7035 \text {, } \\
& \bar{Z}_{2}^{\zeta}=\bar{Z}_{2}^{\xi}=1.0299 \text {, } \\
& \underline{\underline{Z_{2}}}=\underline{\underline{Z_{2}}}+t_{2}\left(\overline{Z_{2}}-\underline{\underline{Z_{2}}}\right)=0.7035+t_{2} \\
& \xi_{2}\left(Z_{2}(x)\right)= \begin{cases}1, & \text { if } Z_{2}(x)<0.7035 \\
1-\frac{Z_{2}(x)-0.64}{1.1391-0.64}, & \text { if } 0.7035 \leq Z_{2}(x) \leq 1.0299 \\
0, & \text { if } Z_{2}(x)>1.01299\end{cases} \\
& \psi_{2}\left(Z_{2}(x)\right)= \begin{cases}1, & \text { if } Z_{2}(x)<0.7035, \\
1-\frac{Z_{2}(x)-0.7035}{s_{2}}, & \text { if } 0.7035 \leq Z_{2}(x) \leq 0.7035+s_{2}, \\
0, & \text { if } Z_{2}(x)>0.7035+s_{2},\end{cases} \\
& \zeta_{2}\left(Z_{2}(x)\right)= \begin{cases}1, & \text { if } Z_{2}(x)>1.0299, \\
1-\frac{1.0299-Z_{2}(x)}{1.0299-0.7035-t_{2},} & \text { if } 0.7035+t_{2} \leq Z_{2}(x) \leq 1.0299, \\
0, & \text { if } Z_{2}(x)<0.7035+t_{2} .\end{cases}
\end{aligned}
$$


Step 5: construct the equivalent neutrosophic programming model for problem (18), which is given as follows:

$\operatorname{Max} \alpha-\beta+\gamma$

subject to

$x_{11}+x_{12}+x_{13}+x_{14} \leq 15$,

$x_{21}+x_{22}+x_{23}+x_{24} \leq 25$,

$x_{31}+x_{32}+x_{33}+x_{34} \leq 20$,

$x_{11}+x_{21}+x_{31} \geq 15$,

$x_{12}+x_{22}+x_{32} \geq 25$,

$x_{13}+x_{23}+x_{33} \geq 5$,

$x_{14}+x_{24}+x_{34} \geq 15$,

$\frac{\left(10 x_{11}+14 x_{12}+8 x_{13}+12 x_{14}+8 x_{21}+12 x_{22}+14 x_{23}+8 x_{24}+9 x_{31}+6 x_{32}+15 x_{33}+9 x_{34}\right)}{\left(15 x_{11}+12 x_{12}+16 x_{13}+8 x_{14}+10 x_{21}+6 x_{22}+13 x_{23}+12 x_{24}+13 x_{31}+15 x_{32}+12 x_{33}+10 x_{34}\right)}+0.7105 \alpha \leq 1.3143$,

$\frac{\left(14 x_{11}+9 x_{12}+11 x_{13}+9 x_{14}+12 x_{21}+9 x_{22}+6 x_{23}+15 x_{24}+6 x_{31}+9 x_{32}+12 x_{33}+10 x_{34}\right)}{\left(12 x_{11}+14 x_{12}+7 x_{13}+17 x_{14}+6 x_{21}+11 x_{22}+13 x_{23}+10 x_{24}+9 x_{31}+15 x_{32}+12 x_{33}+16 x_{34}\right)}+0.3264 \alpha \leq 1.1391$,

$\frac{\left(10 x_{11}+14 x_{12}+8 x_{13}+12 x_{14}+8 x_{21}+12 x_{22}+14 x_{23}+8 x_{24}+9 x_{31}+6 x_{32}+15 x_{33}+9 x_{34}\right)}{\left(15 x_{11}+12 x_{12}+16 x_{13}+8 x_{14}+10 x_{21}+6 x_{22}+13 x_{23}+12 x_{24}+13 x_{31}+15 x_{32}+12 x_{33}+10 x_{34}\right)}+s_{1} \gamma-s_{1} \leq 0.6038$

$\frac{\left(14 x_{11}+9 x_{12}+11 x_{13}+9 x_{14}+12 x_{21}+9 x_{22}+6 x_{23}+15 x_{24}+6 x_{31}+9 x_{32}+12 x_{33}+10 x_{34}\right)}{\left(12 x_{11}+14 x_{12}+7 x_{13}+17 x_{14}+6 x_{21}+11 x_{22}+13 x_{23}+10 x_{24}+9 x_{31}+15 x_{32}+12 x_{33}+16 x_{34}\right)}+s_{2} \gamma-s_{2} \leq 0.7035$,

$\left(10 x_{11}+14 x_{12}+8 x_{13}+12 x_{14}+8 x_{21}+12 x_{22}+14 x_{23}+8 x_{24}+9 x_{31}+6 x_{32}+15 x_{33}+9 x_{34}\right.$

$\left(15 x_{11}+12 x_{12}+16 x_{13}+8 x_{14}+10 x_{21}+6 x_{22}+13 x_{23}+12 x_{24}+13 x_{31}+15 x_{32}+12 x_{33}+10 x_{34}\right)+\left(0.7105+t_{1}\right) \beta-t_{1}$

$\leq 0.6038$,

$\frac{\left(14 x_{11}+9 x_{12}+11 x_{13}+9 x_{14}+12 x_{21}+9 x_{22}+6 x_{23}+15 x_{24}+6 x_{31}+9 x_{32}+12 x_{33}+10 x_{34}\right)}{\left(12 x_{11}+14 x_{12}+7 x_{13}+17 x_{14}+6 x_{21}+11 x_{22}+13 x_{23}+10 x_{24}+9 x_{31}+15 x_{32}+12 x_{33}+16 x_{34}\right)}+\left(0.3264+t_{2}\right) \beta-t_{2}$

$\leq 0.7035$

$\alpha \geq \gamma, \alpha \geq \beta, \alpha+\gamma+\beta \leq 3,0 \leq t_{1}, s_{1} \leq 0.7035,0 \leq t_{2}, s_{2} \leq 0.3264$,

$\alpha, \gamma, \beta \in[0,1], x_{i j} \geq 0, \quad i=1,2,3,4, j=1,2,3$.

Step 6: solve neutrosophic model (25) using LINDO software. We have obtained the best compromise solution, which is shown in Table 2.

5.1. Case Study Problem. In this work, consider that a wind turbine manufacture company has four different plants in different countries. And the company sells its wind turbine blades to four different countries. All plants manufactured the same type of wind turbine blades. However, the wind turbine blade is sold at different prices in different countries due to some factors. The $i^{\text {th }}$ source can provide $a_{i}$ units of a certain product, and the $j^{\text {th }}$ destination has a demand for $b_{j}$ units of the same product. Thus, when selling wind turbine blades to different countries, the manufacturing company presents three main objectives; that is, it considers minimizing the total cost, minimizing the total delivery time, and minimizing deterioration. Hence, the objectives are to minimize the actual cost/standard cost, actual deterioration/ standard deterioration, and actual delivery time/standard delivery time involved in transporting wind turbine blades from various source points to several destinations. The 
TABLE 2: Comparison between the proposed NGP method with GP, FGP, and IFGP methods for the example problem.

\begin{tabular}{lcccc}
\hline DVs & GP & FGP & IFGP & Proposed method (NGP) \\
\hline$x_{11}$ & 0 & 5 & 5 & 0 \\
$x_{12}$ & 12 & 5 & 5 & 3 \\
$x_{13}$ & 3 & 0 & 5 & 0 \\
$x_{14}$ & 0 & 5 & 0 & 12 \\
$x_{21}$ & 11 & 0 & 10 & 15 \\
$x_{22}$ & 11 & 5 & 0 & 2 \\
$x_{23}$ & 1 & 9 & 0 & 5 \\
$x_{24}$ & 1 & 0 & 15 & 3 \\
$x_{31}$ & 4 & 20 & 0 & 0 \\
$x_{32}$ & 2 & 0 & 0 & 20 \\
$x_{33}$ & 1 & 0 & 0 \\
$x_{34}$ & 14 & 0.7805 & 0 \\
$Z_{1}$ & 1.0366 & 1.0296 & 0.7321 \\
$Z_{2}$ & 0.8374 & 0.7534 & 0.7829 \\
\hline
\end{tabular}

restrictions that are being active are the limited availability at the source points and the minimum demand that is to be satisfied at the destinations.

\subsubsection{Objective Functions}

(1) Minimize Total Transportation Costs. The first objective $\left(Z_{1}\right)$ is modeled for minimizing the total transportation cost of wind turbine blades from source $i$ to destination $j$. This is because, basically, wind turbine blades have a very wide width, huge height, and heavy weight. Therefore, these blades are difficult to carry by road, rail, and more. Furthermore, the transportation cost of such blades is calculated depending on the source and destination.

$\operatorname{Min} Z_{1}=\frac{\text { transportation actual cost }\left(C_{a}\right)}{\text { transportation standard cost }\left(C_{s}\right)}=\frac{\sum_{i=1}^{m} \sum_{j=1}^{n} n_{i j}^{(1)} x_{i j}}{\sum_{i=1}^{m} \sum_{j=1}^{n} d_{i j}^{(1)} x_{i j}}$.

(2) Minimize Total Deterioration. The second objective is constructed for minimizing the product total deterioration of blades from source $i$ to destination $j$.

$$
\operatorname{Min} Z_{2}=\frac{\text { actual deterioration }\left(D_{a}\right)}{\text { standard deterioration }\left(D_{s}\right)}=\frac{\sum_{i=1}^{m} \sum_{j=1}^{n} n_{i j}^{(2)} x_{i j}}{\sum_{i=1}^{m} \sum_{j=1}^{n} d_{i j}^{(2)} x_{i j}} .
$$

(3) Minimize Total Delivery Time. The third objective $Z_{3}$ is formulated for minimizing the total delivery time of wind turbine blades from source $i$ to destination $j$. Due to the dimension of the wind turbine plate, the travel time from the source to the destination is carried out carefully.

$$
\operatorname{Min} Z_{3}=\frac{\text { delivery actual time }\left(T_{a}\right)}{\text { delivery standard time }\left(T_{s}\right)}=\frac{\sum_{i=1}^{m} \sum_{j=1}^{n} n_{i j}^{(3)} x_{i j}}{\sum_{i=1}^{m} \sum_{j=1}^{n} d_{i j}^{(3)} x_{i j}} .
$$

In this work, a wind turbine blade transportation problem optimizes with the neutrosophic goal programming model as the fractional transportation problem with multiple objectives. Therefore, the constraints are presumed to be crisp ones. Significantly, the MOFTP model described above has an optimal solution only if the total supply available at all sources is equal to the total demand for all destinations.

5.1.2. Description of the Problem and Data. A wind turbine production company sells its wind turbine blades from source $i(i=1,2,3,4)$ to destination $j(j=1,2,3,4)$. For some reasons, the same type of wind turbine blades is sold at different prices in different countries.

The total actual cost $\left(C_{a}\right)$ and the standard cost $\left(C_{s}\right)$ are

$$
\begin{aligned}
C_{a} & =\left[\begin{array}{llll}
20 & 18 & 18 & 13 \\
19 & 13 & 16 & 18 \\
15 & 11 & 17 & 12 \\
14 & 14 & 16 & 13
\end{array}\right], \\
C_{s} & =\left[\begin{array}{llll}
18 & 16 & 19 & 12 \\
20 & 15 & 15 & 18 \\
16 & 12 & 15 & 10 \\
13 & 12 & 16 & 14
\end{array}\right] .
\end{aligned}
$$

The total actual time $\left(T_{a}\right)$ and the standard time $\left(T_{s}\right)$ are

$$
\begin{aligned}
& T_{a}=\left[\begin{array}{llll}
30 & 34 & 34 & 34 \\
26 & 24 & 31 & 29 \\
21 & 20 & 26 & 29 \\
21 & 22 & 25 & 31
\end{array}\right], \\
& T_{s}=\left[\begin{array}{llll}
28 & 33 & 32 & 35 \\
25 & 25 & 32 & 28 \\
22 & 20 & 25 & 28 \\
21 & 20 & 26 & 30
\end{array}\right] .
\end{aligned}
$$

The total actual deterioration $\left(D_{a}\right)$ and the standard deterioration $\left(D_{s}\right)$ are 


$$
\begin{aligned}
& D_{a}=\left[\begin{array}{llll}
40 & 34 & 37 & 28 \\
38 & 28 & 37 & 40 \\
42 & 39 & 30 & 41 \\
29 & 38 & 32 & 32
\end{array}\right], \\
& D_{s}=\left[\begin{array}{llll}
38 & 35 & 35 & 32 \\
38 & 30 & 34 & 36 \\
40 & 36 & 32 & 37 \\
33 & 35 & 32 & 32
\end{array}\right] .
\end{aligned}
$$

The supplies $\left(a_{i}\right)$ and demands $\left(b_{i}\right)$ are

$$
\begin{aligned}
a_{i} & =\left[\begin{array}{llll}
25 & 30 & 32 & 28
\end{array}\right], \\
b_{i} & =\left[\begin{array}{llll}
10 & 14 & 22 & 18
\end{array}\right] .
\end{aligned}
$$

$$
\begin{aligned}
& \operatorname{Min} Z_{1}=\frac{20 x_{11}+18 x_{12}+18 x_{13}+13 x_{14}+19 x_{21}+13 x_{22}+16 x_{23}+18 x_{24}+15 x_{31}+11 x_{32}+17 x_{33}+12 x_{34}+14 x_{41}+14 x_{42}+16 x_{43}+13 x_{44}}{18 x_{11}+16 x_{12}+19 x_{13}+12 x_{14}+20 x_{21}+15 x_{22}+15 x_{23}+18 x_{24}+16 x_{31}+12 x_{32}+15 x_{33}+10 x_{34}+13 x_{41}+12 x_{42}+16 x_{43}+14 x_{44}}, \\
& \operatorname{Min} Z_{2}=\frac{30 x_{11}+34 x_{12}+34 x_{13}+34 x_{14}+26 x_{21}+24 x_{22}+31 x_{23}+29 x_{24}+21 x_{31}+20 x_{32}+26 x_{33}+29 x_{34}+21 x_{41}+22 x_{42}+25 x_{43}+31 x_{44}}{28 x_{11}+33 x_{12}+32 x_{13}+35 x_{14}+25 x_{21}+25 x_{22}+32 x_{23}+28 x_{24}+22 x_{31}+20 x_{32}+25 x_{33}+28 x_{34}+21 x_{41}+20 x_{42}+26 x_{43}+30 x_{44}}, \\
& \operatorname{Min} Z_{3}=\frac{40 x_{11}+34 x_{12}+37 x_{13}+28 x_{14}+38 x_{21}+28 x_{22}+37 x_{23}+40 x_{24}+42 x_{31}+39 x_{32}+30 x_{33}+41 x_{34}+29 x_{41}+38 x_{42}+32 x_{43}+32 x_{44}}{38 x_{11}+35 x_{12}+35 x_{13}+32 x_{14}+38 x_{21}+30 x_{22}+34 x_{23}+36 x_{24}+40 x_{31}+36 x_{32}+32 x_{33}+37 x_{34}+33 x_{41}+35 x_{42}+32 x_{43}+32 x_{44}}, \\
& \text { subject to } \\
& x_{11}+x_{12}+x_{13}+x_{14} \leq 25 \\
& x_{21}+x_{22}+x_{23}+x_{24} \leq 30 \\
& x_{31}+x_{32}+x_{33}+x_{34} \leq 32 \\
& x_{41}+x_{42}+x_{43}+x_{44} \leq 28 \\
& x_{11}+x_{21}+x_{31}+x_{41} \leq 22 \\
& x_{12}+x_{22}+x_{32}+x_{42} \leq 24 \\
& x_{13}+x_{23}+x_{33}+x_{43} \leq 32 \\
& x_{14}+x_{24}+x_{34}+x_{44} \leq 37 \\
& x_{i j} \geq 0, \quad i=1,2,3,4, j=1,2,3,4
\end{aligned}
$$

5.1.3. Solution Procedure. In this section, the fractional transportation problem will optimize three objectives simultaneously with a set of constraints. The mathematical model of the above problem is as follows:

Problem (18) is solved using the neutrosophic mathematical programming approach in the following steps:

Step 1: solve the above model in each objective function individually as a single-objective transportation problem subject to the same constraints (33) and the individual solution as $X_{1}=(0,0,25,0,0,14,7,9,22,10$, $0,0,0,0,0,28), X_{2}=(0,0,0,25,0,24,4,2,22,0,0,10,0$, $0,28,0)$, and $X_{3}=(0,0,0,25,0,24,0,6,0,0,32,0,22$, $0,0,6)$.

Step 2: construct the payoff matrix by evaluating three objectives with the obtained three solutions.

$$
\left.\begin{array}{c|ccc} 
& Z_{1} & Z_{2} & Z_{3} \\
Z_{1} & 0.9416 & 1.0139 & 1.0383 \\
Z_{2} & 0.9873 & 0.9711 & 0.9886 \\
Z_{3} & 1.0352 & 0.9983 & 0.9249
\end{array}\right)
$$

Step 3: compute the lower and upper bounds for all objectives with the payoff matrix, which are assigned using the following formula:

$$
\begin{aligned}
& \overline{Z_{k}}=\max \left\{Z_{k}(x)\right\}_{k=1}^{3}, \\
& \underline{Z_{k}}=\min \left\{Z_{k}(x)\right\}_{k=1}^{3} .
\end{aligned}
$$

The bounds of each objective function are defined by

$$
\begin{aligned}
& 0.9416 \leq Z_{1} \leq 1.0352, \\
& 0.9711 \leq Z_{2} \leq 1.0139 \\
& 0.9249 \leq Z_{3} \leq 1.0383
\end{aligned}
$$

Step 4: define all membership functions based on the neutrosophic concept for three objectives with equations (11)-(13). 
For the first objective $Z_{1}$ :

$$
\begin{aligned}
& \bar{Z}_{1}^{\xi}=1.035228, \underline{Z_{1}}=0.9416, \\
& \bar{Z}_{1}^{\psi}=\underline{Z_{1}}+S_{1}\left(\overline{Z_{1}}-\underline{\underline{Z_{1}}}\right)=0.9416+s_{1}, \underline{Z_{1}}=\underline{\underline{Z_{1}}}=0.9416, \\
& \bar{Z}_{1}^{\zeta}=\bar{Z}_{1}^{\xi}=1.0352, \underline{\underline{Z_{1}}}=\underline{Z_{1}}+t_{1}\left(\bar{Z}_{1}^{\xi}-\underline{\underline{Z_{1}}}\right)=0.9416+t_{1} .
\end{aligned}
$$

Truth, indeterminacy, and falsity membership func-

tions of the first objective $Z_{1}$ are

$$
\begin{aligned}
& \xi_{1}\left(Z_{1}(x)\right)= \begin{cases}1, & \text { if } Z_{1}(x)<0.9416, \\
1-\frac{Z_{1}(x)-0.9416}{1.0352-0.9416}, & \text { if } 0.9416 \leq Z_{1}(x) \leq 1.0352, \\
0, & \text { if } Z_{1}(x)>1.0352\end{cases} \\
& \psi_{1}\left(Z_{1}(x)\right)= \begin{cases}1, & \text { if } Z_{1}(x)<0.9416, \\
1-\frac{Z_{1}(x)-0.9416}{s_{1}}, & \text { if } 0.9416 \leq Z_{1}(x) \leq 0.9416+s_{1}, \\
0, & \text { if } Z_{1}(x)>0.9416+s_{1},\end{cases} \\
& \zeta_{1}\left(Z_{1}(x)\right)= \begin{cases}1, & \text { if } Z_{1}(x)>1.0352, \\
1-\frac{1.0352-Z_{1}(x)}{1.0352-0.9416-t_{1}}, & \text { if } 0.9416+t_{1} \leq Z_{1}(x) \leq 1.0352, \\
0, & \text { if } Z_{1}(x)<0.9416+t_{1} .\end{cases}
\end{aligned}
$$

For the second objective $Z_{2}$ :

$$
\begin{aligned}
& \bar{Z}_{2}^{\xi}=1.0139, \underline{Z_{2}}=0.9711, \\
& \bar{Z}_{2}^{\psi}=\underline{Z_{2}}+s_{2}\left(\bar{Z}_{2}^{\xi}-\underline{\underline{Z_{2}}}\right)=0.9711+s_{2}, \underline{Z_{2}}=\underline{\underline{Z_{2}}}=0.9711, \\
& \bar{Z}_{2}^{\zeta}={\overline{Z_{2}}}^{\xi}=1.0139, \underline{\underline{Z}} \underline{\underline{Z}}=\underline{Z_{2}}+t_{2}\left(\bar{Z}_{2}^{\xi}-\underline{\underline{Z}}\right)=0.9711+t_{2} .
\end{aligned}
$$


Truth, indeterminacy, and falsity membership functions of the second objective $Z_{2}$ are

$$
\begin{aligned}
& \xi_{2}\left(Z_{2}(x)\right)= \begin{cases}1, & \text { if } Z_{2}(x)<0.9711, \\
1-\frac{Z_{2}(x)-0.9711}{1.0139-0.9711}, & \text { if } 0.9711 \leq Z_{2}(x) \leq 1.0139 \\
0, & \text { if } Z_{2}(x)>1.0139\end{cases} \\
& \psi_{2}\left(Z_{2}(x)\right)= \begin{cases}1, & \text { if } Z_{2}(x)<0.9711, \\
1-\frac{Z_{2}(x)-0.9711}{s_{2}}, & \text { if } 0.9711 \leq Z_{2}(x) \leq 0.9711+s_{2}, \\
0, & \text { if } Z_{2}(x)>0.9711+s_{2},\end{cases} \\
& \zeta_{2}\left(Z_{2}(x)\right)= \begin{cases}1, & \text { if } Z_{2}(x)>1.0139 \\
1-\frac{1.0139-Z_{2}(x)}{1.0139-0.9711-t_{2}}, & \text { if } 0.9711+t_{2} \leq Z_{2}(x) \leq 1.0139 \\
0, & \text { if } Z_{2}(x)<0.9711+t_{2} .\end{cases}
\end{aligned}
$$

For the third objective $Z_{3}$ :

$$
\begin{aligned}
& \bar{Z}^{\xi}=1.0383, \underline{Z_{3}^{\xi}}=0.9249, \\
& \bar{Z}_{3}^{\psi}=\underline{Z_{3}}+s_{3}\left(\overline{Z_{3}^{\xi}}-\underline{\underline{Z_{3}}}\right)=0.9249+s_{3}, \underline{Z_{3}}=\underline{Z_{3}}=0.9249, \\
& \bar{Z}_{3}^{\zeta}=\bar{Z}_{3}^{\xi}=1.0383, \underline{\underline{Z_{3}}}=\underline{Z_{3}}+t_{3}\left(\bar{Z}_{3}^{\xi}-\underline{Z_{3}}\right)=0.9249+t_{3} .
\end{aligned}
$$

Truth, indeterminacy, and falsity membership functions of the third objective $Z_{3}$ are

$$
\begin{aligned}
& \xi_{3}\left(Z_{3}(x)\right)= \begin{cases}1, & \text { if } Z_{3}(x)<0.9249, \\
1-\frac{Z_{3}(x)-0.9249}{1.0383-0.9249}, & \text { if } 0.9249 \leq Z_{3}(x) \leq 1.0383, \\
0, & \text { if } Z_{3}(x)>1.0383,\end{cases} \\
& \psi_{3}\left(Z_{3}(x)\right)= \begin{cases}1, & \text { if } Z_{3}(x)<0.9249, \\
1-\frac{Z_{3}(x)-0.9249}{s_{3}}, & \text { if } 0.9249 \leq Z_{3}(x) \leq 0.9249+S_{3}, \\
0, & \text { if } Z_{3}(x)>0.9249+s_{3},\end{cases} \\
& \zeta_{3}\left(Z_{3}(x)\right)= \begin{cases}1, & \text { if } Z_{3}(x)>1.0383, \\
1-\frac{1.0383-Z_{3}(x)}{1.0383-+0.9249-t_{3},} & \text { if } 0.9249+t_{3} \leq Z_{3}(x) \leq 1.0383, \\
0, & \text { if } Z_{3}(x)<0.9249+t_{3} .\end{cases}
\end{aligned}
$$


Step 5: construct the equivalent neutrosophic programming model for problem (33), which is given as follows:

$\operatorname{Max} \alpha-\beta+\gamma$

subject to

$x_{11}+x_{12}+x_{13}+x_{14}=25$

$x_{21}+x_{22}+x_{23}+x_{24}=30$

$x_{31}+x_{32}+x_{33}+x_{34}=32$,

$x_{41}+x_{42}+x_{43}+x_{44}=28$,

$x_{11}+x_{21}+x_{31}+x_{41}=22$,

$x_{12}+x_{22}+x_{32}+x_{42}=24$,

$x_{13}+x_{23}+x_{33}+x_{43}=32$,

$x_{14}+x_{24}+x_{34}+x_{44}=37$,

$\frac{\left(\begin{array}{c}20 x_{11}+18 x_{12}+18 x_{13}+13 x_{14}+19 x_{21}+13 x_{22}+16 x_{23}+18 x_{24}+15 x_{31} \\ +11 x_{32}+17 x_{33}+12 x_{34}+14 x_{41}+14 x_{42}+16 x_{43}+13 x_{44}\end{array}\right)}{\left(18 x_{11}+16 x_{12}+19 x_{13}+12 x_{14}+20 x_{21}+15 x_{22}+15 x_{23}+18 x_{24}+16 x_{31}+12 x_{32}+15\right.}$

$\frac{\left(\begin{array}{c}18 x_{11}+16 x_{12}+19 x_{13}+12 x_{14}+20 x_{21}+15 x_{22}+15 x_{23}+18 x_{24}+16 x_{31}+12 x_{32}+15 x_{33} \\ +10 x_{34}+13 x_{41}+12 x_{42}+16 x_{43}+14 x_{44}\end{array}\right)}{(0.0935 \alpha \leq 1.0352,}$

$\frac{\left(\begin{array}{c}30 x_{11}+34 x_{12}+34 x_{13}+34 x_{14}+26 x_{21}+24 x_{22}+31 x_{23}+29 x_{24}+21 x_{31} \\ +20 x_{32}+26 x_{33}+29 x_{34}+21 x_{41}+22 x_{42}+25 x_{43}+31 x_{44}\end{array}\right)}{\left(\begin{array}{c}28 x_{11}+33 x_{12}+32 x_{13}+35 x_{14}+25 x_{21}+25 x_{22}+32 x_{23}+28 x_{24}+22 x_{31}+20 x_{32}+25 x_{33} \\ +28 x_{34}+21 x_{41}+20 x_{42}+26 x_{43}+30 x_{44}\end{array}\right)}+0.0428 \alpha \leq 1.0139$

$\frac{\left(\begin{array}{c}40 x_{11}+34 x_{12}+37 x_{13}+28 x_{14}+38 x_{21}+28 x_{22}+37 x_{23}+40 x_{24}+42 x_{31} \\ +39 x_{32}+30 x_{33}+41 x_{34}+29 x_{41}+38 x_{42}+32 x_{43}+32 x_{44}\end{array}\right)}{\left(\begin{array}{c}38 x_{11}+35 x_{12}+35 x_{13}+32 x_{14}+38 x_{21}+30 x_{22}+34 x_{23}+36 x_{24}+40 x_{31}+36 x_{32}+32 x_{33} \\ +37 x_{34}+33 x_{41}+35 x_{42}+32 x_{43}+32 x_{44}\end{array}\right)}+0.1134 \alpha \leq 1.038317$,

$\left(\begin{array}{c}20 x_{11}+18 x_{12}+18 x_{13}+13 x_{14}+19 x_{21}+13 x_{22}+16 x_{23}+18 x_{24}+15 x_{31}+11 x_{32}+17 x_{33}+12 x_{34} \\ +14 x_{41}+14 x_{42}+16 x_{43}+13 x_{44}\end{array}\right)$

$\left(\begin{array}{c}18 x_{11}+16 x_{12}+19 x_{13}+12 x_{14}+20 x_{21}+15 x_{22}+15 x_{23}+18 x_{24}+16 x_{31}+12 x_{32}+15 x_{33} \\ +10 x_{34}+13 x_{41}+12 x_{42}+16 x_{43}+14 x_{44}\end{array}\right)(x)$

$+s_{1} \gamma-s_{1} \leq 0.9416$ 


$$
\begin{aligned}
& \frac{\left(\begin{array}{c}
30 x_{11}+34 x_{12}+34 x_{13}+34 x_{14}+26 x_{21}+24 x_{22}+31 x_{23}+29 x_{24}+21 x_{31}+20 x_{32}+26 x_{33}+29 x_{34} \\
+21 x_{41}+22 x_{42}+25 x_{43}+31 x_{44}
\end{array}\right)}{28 x_{11}+33 x_{12}+32 x_{13}+35 x_{14}+25 x_{21}+25 x_{22}}+s_{2} \gamma \\
& \left(\begin{array}{l}
+32 x_{23}+28 x_{24}+22 x_{31}+20 x_{32}+25 x_{33}+28 x_{34}+21 x_{41}+20 x_{42}+26 x_{43}+30 x_{44}
\end{array}\right) \\
& -s_{2} \leq 0.9711,
\end{aligned}
$$$$
\begin{aligned}
& \frac{\left(\begin{array}{c}
40 x_{11}+34 x_{12}+37 x_{13}+28 x_{14}+38 x_{21}+28 x_{22}+37 x_{23}+40 x_{24} \\
+42 x_{31}+39 x_{32}+30 x_{33}+41 x_{34}+29 x_{41}+38 x_{42}+32 x_{43}+32 x_{44}
\end{array}\right)}{\left(\begin{array}{c}
38 x_{11}+35 x_{12}+35 x_{13}+32 x_{14}+38 x_{21}+30 x_{22}+34 x_{23}+36 x_{24}+40 x_{31}+36 x_{32}+32 x_{33}+37 x_{34}+33 x_{41} \\
+35 x_{42}+32 x_{43}+32 x_{44}
\end{array}\right)} \\
& +s_{3} \gamma-s_{3} \leq 0.9249, \\
& \frac{\left(\begin{array}{c}
20 x_{11}+18 x_{12}+18 x_{13}+13 x_{14}+19 x_{21}+13 x_{22}+16 x_{23}+18 x_{24}+15 x_{31}+11 x_{32}+17 x_{33} \\
+12 x_{34}+14 x_{41}+14 x_{42}+16 x_{43}+13 x_{44}
\end{array}\right)}{18 x_{11}+16 x_{12}+19 x_{13}+12 x_{14}+20 x_{21}}-\left(0.0936-t_{1}\right) \beta-t_{1} \\
& \left(\begin{array}{c}
+15 x_{22}+15 x_{23}+18 x_{24}+16 x_{31}+12 x_{32}+15 x_{33}+10 x_{34}+13 x_{41}+12 x_{42}+16 x_{43}+14 x_{44}
\end{array}\right)
\end{aligned}
$$

$\leq 0.9416$,

$$
\begin{aligned}
& \frac{\left(\begin{array}{c}
30 x_{11}+34 x_{12}+34 x_{13}+34 x_{14}+26 x_{21}+24 x_{22}+31 x_{23} \\
+29 x_{24}+21 x_{31}+20 x_{32}+26 x_{33}+29 x_{34}+21 x_{41}+22 x_{42}+25 x_{43}+31 x_{44}
\end{array}\right)}{\left(\begin{array}{c}
28 x_{11}+33 x_{12}+32 x_{13}+35 x_{14}+25 x_{21}+25 x_{22}+32 x_{23}+28 x_{24}+22 x_{31}+20 x_{32}+25 x_{33}+28 x_{34}+21 x_{41} \\
+20 x_{42}+26 x_{43}+30 x_{44}
\end{array}\right)} \\
& -\left(0.0428-t_{2}\right) \beta-t_{2} \leq 0.9711 \text {, } \\
& \frac{\left(\begin{array}{c}
40 x_{11}+34 x_{12}+37 x_{13}+ \\
+41 x_{14}+38 x_{21}+28 x_{22}+37 x_{23}+40 x_{24}+42 x_{31}+39 x_{32}+30 x_{33}
\end{array}\right)}{\left(\begin{array}{c}
38 x_{11}+35 x_{12}+35 x_{13}+32 x_{14}+38 x_{21} \\
+30 x_{22}+34 x_{23}+36 x_{24}+40 x_{31}+36 x_{32}+32 x_{33}+37 x_{34}+33 x_{41}+35 x_{42}+32 x_{43}+32 x_{44}
\end{array}\right)}-\left(0.1134-x_{3}\right) \beta \\
& -t_{3} \leq 0.9249 \alpha \geq \gamma, \\
& \alpha \geq \beta, \alpha+\gamma+\beta \leq 3, \quad \alpha, \gamma, \beta \in[0,1], k=1,2,3 .
\end{aligned}
$$

Step 6: solve neutrosophic model (25) using LINDO software. We have obtained the best compromise solution, which is shown in Table 3.

\section{Results and Discussion}

A numerical example and a case study problem are used to investigate the efficiency of the proposed NGP. In this regard, the neutrosophic model for the MOFTP is constructed based on truth, indeterminacy, and falsity membership functions to obtain the best compromise solution. Then, the NGP problems are solved using LINGO software. The obtained solution is compared with the solutions of GP, FGP, and IFGP methods which is shown in Tables 2 and 3 for the numerical example and case study problems. From Table 2, we observe that the value of the first objective function is $1.0366,0.7534,0.7805$, and 0.7321 for GP, FGP, IFGP, and NGP, respectively. This shows that the NGP approach provides the minimum objective value. Similarly, the second objective function value is $0.8374,0.8655,1.0296$, and 0.7829 for GP, FGP, IFGP, and NGP, respectively. The NGP approach provides the minimum value; this implies that the NGP method gives the best compromise solution. Similary, from Table 3, we see that the NGP method gives the minimum values when compared to the GP, FGP, and IFGP methods. Hence, NGP has a better compromise solution than the GP, FGP, and IFGP approaches for both problems. The proposed approach can be used to solve any 
TABLE 3: Comparison between the proposed NGP with GP, FGP, and IFGP for the case study problem.

\begin{tabular}{lcccc}
\hline DVs & GP & FGP & IFGP & Proposed method (NGP) \\
\hline$x_{11}$ & 5 & 0 & 0 & 0 \\
$x_{12}$ & 0 & 10 & 0 & 0 \\
$x_{13}$ & 4 & 5 & 0 & 3 \\
$x_{14}$ & 10 & 25 & 22 \\
$x_{21}$ & 16 & 8 & 21 & 4 \\
$x_{22}$ & 8 & 6 & 7 & 24 \\
$x_{23}$ & 6 & 1 & 0 & 0 \\
$x_{24}$ & 11 & 1 & 4 & 18 \\
$x_{31}$ & 5 & 0 & 20 & 0 \\
$x_{32}$ & 8 & 0 & 0 & 14 \\
$x_{33}$ & 7 & 25 & 4 & 0 \\
$x_{34}$ & 17 & 1 & 8 & 0 \\
$x_{41}$ & 0 & 0 & 0 \\
$x_{42}$ & 1 & 26 & 28 & 13 \\
$x_{43}$ & 11 & 1 & 0 & 15 \\
$x_{44}$ & 0 & 1.0196 & 0.9875 & 0.9789 \\
$Z_{1}$ & 16 & 1.032 & 0.9748 & 0.9874 \\
$Z_{2}$ & 1.0230 & 1.0248 & 0.9842 & 0.9601 \\
$Z_{3}$ & 1.0085 & 0 & \\
\hline
\end{tabular}

deterministic multiobjective optimization problem efficiently. Both nonpreference compromise solutions and preference-based solutions can be generated using the proposed method. Overall, the proposed strategy is better suited to problems involving multiobjective structures. Furthermore, this approach can be extended to solve optimization problems with uncertain parameters.

\section{Conclusion}

A neutrosophic programming framework is developed in this study to obtain an optimal compromise solution for the MOFTP. To address the problem, the membership functions of degrees of truth, falsity, and indeterminacy are defined, and a compromise optimization model has been created based on Bellman and Zadeh's min operator. The efficiency of the proposed method has been illustrated through a numerical example and a case study problem. Finally, this study shows that the results obtained from the proposed method are superior to the existing methods such as GP, FGP, and IFGP. Among the approaches, the NGP method achieves a better optimal compromise solution, which is also true for the optimal solution obtained via the global criterion method. The NGP technique also generates a selection of optimal compromise solutions from which the decision maker can select the desired answer. The key advantages of the NGP approach over the other methods have been discussed. The primary contribution of the proposed study has been explained as follows.

The MOFTP model was created to help decision makers overcome the challenges they face when making decisions in real life. The MOFTP was solved using the NGP technique in this study. The truth, indeterminacy, and falsity membership functions have been introduced into the MOFTP to handle the neutrosophic environment. Four different methods, such as the goal programming method, fuzzy programming method, intuitionistic fuzzy programming method, and neutrosophic goal programming, have been utilized to derive the optimal compromise solution from the deterministic MOFTP. A comparison study has been conducted between the best compromise solutions derived from each of the four methodologies.

The major limitation of the proposed method is that if all objectives are of minimization type, then the proposed approach provides the best solution. When the conflicting objective occurs, the proposed method may or may not provide the best solution. The future research direction is to consider the nonlinear membership function such as exponential and hyperbolic instead of the linear membership function for solving the MOFTP. Furthermore, the proposed method can be extended for solving optimization problems that involve fuzzy, intuitionistic fuzzy, and neutrosophic parameters and variables.

\section{Data Availability}

No data were used to support the findings of this study.

\section{Conflicts of Interest}

The authors declare that they have no conflicts of interest.

\section{Authors' Contributions}

All authors contributed equally and significantly in conducting this research work and writing this paper.

\section{References}

[1] F. L. Hitchcock, "The distribution of a product from several sources to numerous localities," Journal of Mathematics and Physics, vol. 20, no. 1-4, pp. 224-230, 1941.

[2] G. B. Dantzig and M. N. Thapa, Linear Programming 2: Theory and Extensions, Springer, New York, NY, USA, 2006.

[3] I. D. Ezekiel and S. O. Edeki, "Modified Vogel approximation method for balanced transportation models towards optimal 
option settings," International Journal of Civil Engineering \& Technology, vol. 9, pp. 358-366, 2018.

[4] B. Amaliah, C. Fatichah, and E. Suryani, "A new heuristic method of finding the initial basic feasible solution to solve the transportation problem," Journal of King Saud UniversityComputer and Information Sciences, 2020.

[5] K. Karagul and Y. Sahin, "A novel approximation method to obtain initial basic feasible solution of transportation problem," Journal of King Saud University-Engineering Sciences, vol. 32, no. 3, pp. 211-218, 2020.

[6] M. L. Sang and L. J. Moore, "Optimizing transportation problems with multiple objectives," AIIE Transactions, vol. 5, no. 4 , pp. 333-338, 1973.

[7] W. Edwards, "How to use multiattribute utility measurement for social decisionmaking," IEEE Transactions on Systems, Man, and Cybernetics, vol. 7, no. 5, pp. 326-340, 1977.

[8] H. J. Einhorn and W. McCoach, "A simple multiattribute utility procedure for evaluation," Behavioral Science, vol. 22, no. 4, pp. 270-282, 1977.

[9] J. Pratihar, R. Kumar, S. A. Edalatpanah, and A. Dey, "Modified Vogel's approximation method for transportation problem under uncertain environment," Complex \& Intelligent Systems, vol. 7, no. 1, pp. 29-40, 2021.

[10] J. L. Ringuest and D. B. Rinks, "Interactive solutions for the linear multiobjective transportation problem," European Journal of Operational Research, vol. 32, no. 1, pp. 96-106, 1987.

[11] A. K. Bit, M. P. Biswal, and S. S. Alam, "Fuzzy programming approach to multicriteria decision making transportation problem," Fuzzy Sets and Systems, vol. 50, no. 2, pp. 135-141, 1992.

[12] W. F. A. El-Wahed, “A multi-objective transportation problem under fuzziness," Fuzzy Sets and Systems, vol. 117, no. 1, pp. 27-33, 2001.

[13] R. Kumar, S. A. Edalatpanah, S. Jha, and R. Singh, "A Pythagorean fuzzy approach to the transportation problem," Complex \& Intelligent Systems, vol. 5, no. 2, pp. 255-263, 2019.

[14] L. Li and K. K. Lai, "A fuzzy approach to the multiobjective transportation problem," Computers and Operations Research, vol. 27, no. 1, pp. 43-57, 1999.

[15] C. Veeramani and M. Sumathi, "Fuzzy mathematical programming approach for solving fuzzy linear fractional programming problem," RAIRO-Operations Research, vol. 48, no. 1, pp. 109-122, 2014.

[16] R. Verma, M. P. Biswal, and A. Biswas, "Fuzzy programming technique to solve multi-objective transportation problems with some non-linear membership functions," Fuzzy Sets and Systems, vol. 91, no. 1, pp. 37-43, 1997.

[17] T. Beaula and M. Priyadharsini, "A new algorithm for finding a fuzzy optimal solution for intuitionistic fuzzy transportation problems," International Journal of Applications of Fuzzy Sets and Artificial Intelligence, vol. 5, pp. 183-192, 2015.

[18] G. Gourav and A. Kumari, "An efficient method for solving intuitionistic fuzzy transportation problem of type-2," International Journal of Applied and Computational Mathematics, vol. 3, no. 4, pp. 3795-3804, 2017.

[19] C. Veeramani, M. J. Robinson, and S. Vasanthi, "Value- and ambiguity-based approach for solving intuitionistic fuzzy transportation problem with total quantity discounts and incremental quantity discounts," Mathematical Problems in Engineering, vol. 2020, Article ID 8891713, 21 pages, 2020.

[20] M. H. Lohgaonkar and V. H. Bajaj, "Fuzzy approach to solve multi-objective capacitated transportation problem,"
International Journal of Bioinformatics Research, vol. 2, no. 1, pp. 10-14, 2010.

[21] N. Gupta and A. Bari, "Fuzzy multi-objective capacitated transportation problem with mixed constraints," Journal of Statistics Applications \& Probability Letters, vol. 3, no. 2, pp. 1-9, 2014.

[22] M. A. Nomani, I. Ali, and A. Ahmed, "A new approach for solving multi-objective transportation problems," International Journal of Management Science and Engineering Management, vol. 12, no. 3, pp. 1-9, 2016.

[23] S. Pramanik and D. Banerjee, "Multi-objective chance constrained capacitated transportation problem based on fuzzy goal programming," International Journal of Computer Application, vol. 44, no. 20, pp. 42-46, 2012.

[24] M. Jain and P. K. Saksena, "Time minimizing transportation problem with fractional bottleneck objective function," Yugoslav Journal of Operations Research, vol. 22, no. 1, pp. 115-129, 2012.

[25] S. Sadia, N. Gupta, and Q. M. Ali, "Multiobjective capacitated fractional transportation problem with mixed constraints," Mathematical Sciences Letters, vol. 5, no. 3, pp. 235-242, 2016.

[26] J. P. Costa, "Computing non-dominated solutions in MOLFP," European Journal of Operational Research, vol. 181, no. 3, pp. 1464-1475, 2007.

[27] K. T. Atanassov, "Intuitionistic fuzzy sets," in Physica, pp. 1-137, Springer, Heidelberg, Germany, 1999.

[28] I. Deli and N. Çağman, "Intuitionistic fuzzy parameterized soft set theory and its decision making," Applied Soft Computing, vol. 28, no. 4, pp. 109-113, 2015.

[29] M. Abdel-Basset, G. Manogaran, A. Gamal, and F. Smarandache, "A hybrid approach of neutrosophic sets and DEMATEL method for developing supplier selection criteria," Design Automation for Embedded Systems, vol. 22, no. 3, pp. 257-278, 2018.

[30] A. H. Nafei and S. H. Nasseri, "A new approach for solving neutrosophic integer programming problems," International Journal of Applied Operational Research, vol. 9, pp. 1-9, 2019.

[31] S. A. Edalatpanah, "A nonlinear approach for neutrosophic linear programming," Journal of Applied Research on Industrial Engineering, vol. 6, no. 4, pp. 367-373, 2019.

[32] R. M. Rizk-Allah, A. E. Hassanien, and M. Elhoseny, "A multiobjective transportation model under neutrosophic environment," Computers \& Electrical Engineering, vol. 69, pp. 705-719, 2018.

[33] J. Ye, "Neutrosophic number linear programming method and its application under neutrosophic number environments," Soft Computing, vol. 22, no. 14, pp. 4639-4646, 2018.

[34] F. Ahmad, "Robust neutrosophic programming approach for solving intuitionistic fuzzy multiobjective optimization problems," Complex \& Intelligent Systems, vol. 7, no. 4, pp. 1935-1954, 2021.

[35] H.-J. Zimmermann, "Fuzzy programming and linear programming with several objective functions," Fuzzy Sets and Systems, vol. 1, no. 1, pp. 45-55, 1978.

[36] W. F. Abd El-Wahed and S. M. Lee, "Interactive fuzzy goal programming for multi-objective transportation problems," Omega, vol. 34, no. 2, pp. 158-166, 2006.

[37] F. Smarandache, "A unifying field in logics," Neutrosophy: Neutrosophic Probability, Set and Logic, American Research Press, Rehoboth, DE, USA, 1999.

[38] R. Bellman and L. A. Zadeh, "Decision making in fuzzy environment," Management Science, vol. 17, no. 4, pp. 141-164, 1970. 